\title{
Crystallization and preliminary X-ray analysis of a C-terminal TonB fragment from Escherichia coli
}

Jiri Koedding, ${ }^{\text {a* }}$ Patrick Polzer, ${ }^{\text {a }}$ Frank Killig, a S. Peter Howard, ${ }^{b}$ Kinga Gerber, ${ }^{\text {a }}$ Peter Seige, ${ }^{\text {a }}$ Kay Diederichs $^{\mathrm{a}}$ and Wolfram Welte ${ }^{\mathrm{a}}$

${ }^{a}$ Department of Biology, University of Konstanz, 78457 Konstanz, Germany, and ${ }^{\mathbf{b}}$ Department of Microbiology and Immunology, University of Saskatchewan, Saskatoon,

Saskatchewan S7N 5E5, Canada

Correspondence e-mail: jiri.koedding@uni-konstanz.de

The TonB protein located in the cell wall of Gram-negative bacteria mediates the proton motive force from the cytoplasmic membrane to specific outer membrane transporters. A C-terminal fragment of TonB from Escherichia coli consisting of amino-acid residues 147-239 (TonB-92) has been purified and crystallized. Crystals grew in space group $P 2_{1}$ to dimensions of about $1.0 \times 0.12 \times 0.12 \mathrm{~mm}$. A native data set has been obtained to $1.09 \AA$ resolution.

\section{Introduction}

The cell wall of Gram-negative bacteria consists of two lipid bilayers, the outer membrane and the cytoplasmic membrane, enclosing the peptidoglycan layer. All essential compounds have to be transported across the outer membrane by diffusion or specific transport pathways. Specific transporters such as the iron siderophore receptors FhuA, FepA and FecA or the vitamin $\mathrm{B}_{12}$ transporter BtuB are known to be TonB-dependent as they are connected to the cytoplasmic membrane by the TonB protein. TonB mediates the chemical potential of the proton gradient across the cytoplasmic membrane (proton motive force) to the specific outer membrane receptors. TonB belongs to a protein complex together with ExbB and ExbD (Bradbeer, 1993; Larsen et al., 1999; Postle \& Kadner, 2003), which are both located in the cytoplasmic membrane. TonB consists of 239 amino-acid residues, with the first 33 residues forming a hydrophobic anchor (Postle, 1993) that attaches TonB to the cytoplasmic membrane. The major part of TonB spans the periplasmic space to reach the outer membrane receptors. This function is achieved by a flexible proline-rich region between residues 75 and 107 (Postle \& Skare, 1988) that is not essential for the process of energy transduction (Larsen et al., 1994).

The C-terminal domain of TonB forms the contact to the outer membrane receptor, but almost nothing is known about this interaction. It has been shown that a region of critical importance for this protein-protein interaction is located around amino-acid residue 160 (Günter \& Braun, 1990). The three-dimensional structures of two C-terminal fragments of TonB, TonB-86 (residues 155-239; Chang et al., 2001) and TonB-77 (residues 164-239; Koedding et al., 2004), have already been determined. These two TonB fragments crystallized under different conditions and in different space groups. Despite these differ- ences, both structures are similar and reveal a cylinder-shaped dimer. Each monomer contains three $\beta$-strands and a short $\alpha$-helix arranged in a dimer so that the six $\beta$-strands can build up a large antiparallel $\beta$-sheet. The structure of another energy-transducing protein, TolA from Pseudomonas aeruginosa, has also been solved recently (Witty et al., 2002). TolA belongs to the TolA/Q/R system, a system analogous to the TonB/ExbB/ExbD complex, involved in nutrient import (Braun \& Herrmann, 1993). Despite having a sequence identity of only $24 \%$ (LALIGN server; http:// www.ch.embnet.org/software/LALIGN_form.html), the crystal structure of the periplasmic domain of TolA shows a similar topology, but without dimer formation. The importance of dimer formation for the mechanism of energy transduction is thus not yet understood. It has been shown that $\mathrm{C}$-terminal fragments of TonB with more than 90 amino-acid residues behave as monomers in solution (Koedding et al., 2004). However, Sauter et al. (2003) showed that the periplasmic part of TonB is able to dimerize in vivo. Complex formation between monomeric C-terminal fragments of TonB and FhuA has been observed in vitro (Moeck \& Letellier, 2001). On the other hand, a stoichiometry of 2:1 was recently found for TonB-FhuA complexes in vitro (Khursigara et al., 2004).

Here, we present the expression, purification and crystallization of TonB-92, a C-terminal fragment of TonB from Escherichia consisting of amino-acid residues 149-239. We are currently trying to crystallize TonB-92 using the selenomethionine-substitution method (Doublié, 1997).

\section{Materials and methods}

\subsection{Expression and purification}

The C-terminal fragment of TonB (TonB-92) containing the last 92 amino-acid residues of the TonB protein was overexpressed in E. coli 
Table 1

Crystal data and X-ray data-collection statistics for a native TonB-92 crystal.

Values in parentheses refer to the highest resolution shell.

\begin{tabular}{ll}
\hline Protein concentration $\left(\mathrm{mg} \mathrm{ml}^{-1}\right)$ & 20 \\
Crystallization conditions & $100 \mathrm{~m} M$ imidazole \\
& $\mathrm{pH} 8.0,1.1 M$ \\
& sodium citrate, \\
& $100 \mathrm{~m} M \mathrm{NaCl}$ \\
& $a=22.58, b=49.32$, \\
Unit-cell parameters $\left(\AA{ }^{\circ}\right)$ & $c=72.22, \alpha=90$, \\
& $\beta=97.985, \gamma=90$ \\
Space group & $P 2_{1}$ \\
Resolution $(\AA)$ & $10-1.09(1.10-1.09)$ \\
Wavelength $(\AA)$ & $0.95372(13.0 \mathrm{keV})$ \\
Total measured reflections & 427365 \\
Unique reflections & $65348(1727)$ \\
Completeness $(\%)$ & $99.8(99.0)$ \\
$I / \sigma(I)$ & $11.67(1.71)$ \\
$R_{\text {meas }} \dagger(\%)$ & $8.3(59.2)$
\end{tabular}

$\dagger R_{\text {meas }}$ was calculated according to Diederichs \& Karplus (1997).

BL21(DE3) cells containing the plasmid pTB92. For pTB92 the forward primer was US20 (5'-CAT ATG GTG GCT TCA GGA CCA CGC GCA- $3^{\prime}$ ), creating an NdeI restriction site at the $5^{\prime}$-end of the fragment. The return primer was UR136 (5'-GCT AGT TAT TGC TCA GCG G-3'), which hybridizes to the pET30a vector (Novagen) just downstream of the multiple cloning site and contains a Bpu1102I restriction site. Cloning of the resulting PCR fragment into pCSTonB30 (Howard et al., 2001) created the plasmid pTB92. Cells were grown in $2 \times$ YT tryptone yeast extract supplemented with the antibiotic kanamycin $\left(50 \mathrm{mg} \mathrm{l}^{-1}\right)$ at $310 \mathrm{~K}$ and were induced at $\mathrm{OD}_{600}=0.7$ by the addition of $0.4 \mathrm{~m} M$ IPTG (isopropyl$\beta$-D-thiogalactopyranoside, BioVetra). Expression of the $10.2 \mathrm{kDa}$ protein TonB-92 was maintained at $310 \mathrm{~K}$ for $2 \mathrm{~h}$. The pellets from $4 \times 500 \mathrm{ml}$ cell cultures were resus-

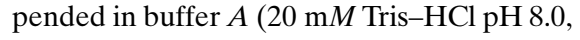
$100 \mathrm{~m} M \mathrm{NaCl}, 1 \mathrm{~m} M$ EDTA) and the cells were broken using a French press $(28 \mathrm{MPa}$;

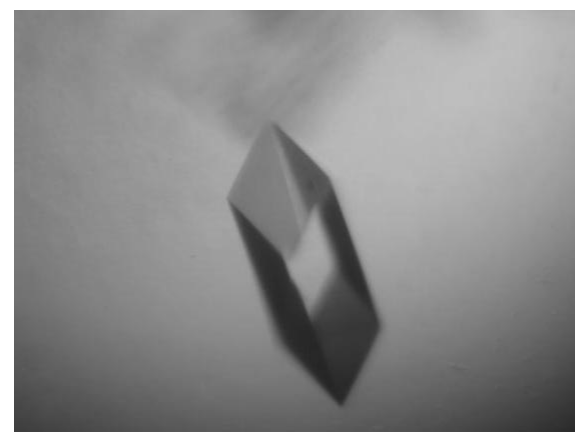

Figure 1

A native TonB-92 crystal of dimensions $1.0 \times 0.12 \times$ $0.12 \mathrm{~mm}$ grown in space group $P 2_{1}$.

Table 2

Results of molecular replacement.

\begin{tabular}{lllc}
\hline & & \multicolumn{2}{l}{ Results of translation search } \\
\cline { 4 - 4 } C-terminal TonB fragment & Results of rotation search $(\mathrm{Rf} / \sigma)$ & Corr & Corr-2 \\
\hline TonB-77 dimer & 3.48 & 0.060 & 0.043 \\
TonB-77 dimer, polyalanine model & 3.53 & 0.081 & 0.117 \\
TonB-77 monomer & 3.48 & 0.062 & 0.117 \\
TonB-77 monomer, polyalanine model & 3.72 & \\
\hline
\end{tabular}

three passes). After centrifugation at $15000 \mathrm{~g}$ for $30 \mathrm{~min}$, the supernatant was loaded onto an SP Sepharose cationexchange column (Amersham Biosciences) and washed with buffer $A$. TonB was eluted from the column with a $\mathrm{NaCl}$ gradient at a salt concentration of about $300 \mathrm{mM} \mathrm{NaCl}$. The eluate was then desalted on a Sephadex G25 column (Amersham Biosciences) before loading onto another strong cationexchange column (Source 15S, Amersham Biosciences). The eluted TonB protein containing about $250 \mathrm{mM} \mathrm{NaCl}$ was again desalted on a Sephadex G25 column with buffer $A$ (without EDTA) and yielded protein at a concentration of $\sim 4 \mathrm{mg} \mathrm{ml}^{-1}$. The mobility of the fragments on $15 \%$ SDSPAGE corresponded to their theoretical molecular weights. The purification was carried out within $1 \mathrm{~d}$ in order to avoid protein degradation. An additional gelfiltration step was added. The protein was concentrated to $10 \mathrm{mg} \mathrm{ml}^{-1}$ (Amicon spincolumn with YMCO 5000) and glycerol was added to a final concentration of $10 \%$. The TonB sample was then loaded onto a gelfiltration column (Superose 12 HR 60/10, Amersham Biosciences) and eluted with buffer $A$.

\subsection{Crystallization and data collection}

For crystallization, the protein sample was concentrated to $20 \mathrm{mg} \mathrm{ml}^{-1}$. Initial screening was performed using Crystal Screen I (Jancarik \& Kim, 1991), Crystal Screen II (Hampton Research) and Wizard Screens I and II (Emerald BioStructures Inc.) at $291 \mathrm{~K}$ in 96-well sitting-drop plates (Hampton Research). Crystals of dimensions $0.3 \times 0.05 \times 0.05 \mathrm{~mm}$ were obtained with Wizard Screen I condition No. 36. Further refinement yielded crystals in 24-well hanging-drop plates (Hampton Research) with $1 \mathrm{ml}$ reservoir solution (100 $\mathrm{m} M$ imidazole $\mathrm{pH}$ 8.0, $1.1 \mathrm{M}$ sodium citrate, $100 \mathrm{~m} M \mathrm{NaCl}$ ) within $5 \mathrm{~d}$ (Fig. 1). The crystallization drop contained $3 \mu \mathrm{l}$ protein solution $\left(20 \mathrm{mg} \mathrm{ml}^{-1}\right)$ and $3 \mu \mathrm{l}$ reservoir solution. Prior to data collection, single crystals were soaked in three different cryoprotectant solutions for $1 \mathrm{~min}$ each and were then transferred into liquid nitrogen. The cryoprotectant solutions contained reservoir solution supplemented with 5,15 and $25 \%$ glycerol. Data collection from the native TonB-92 crystals was carried out to a resolution of $1.09 \AA$ at beamline X06SA at the SLS, Villigen, Switzerland. Raw data were processed using XDS (Kabsch, 1993).

\section{Results and discussion}

We have expressed a C-terminal fragment of E. coli TonB (TonB-92) containing the last 92 amino-acid residues of the protein. TonB92 was purified to near-homogeneity as determined by SDS-PAGE analysis (data not shown) and crystallized at $20 \mathrm{mg} \mathrm{ml}^{-1}$ with the hanging-drop method (Fig. 1). A native data set was collected to $1.09 \AA$ resolution and the raw data were processed with the program $X D S$ (Kabsch, 1993). The space group was determined to be $P 2_{1}$, with two molecules per asymmetric unit (Table 1). Further data-collection statistics are given in Table 1. Molecular replacement with the search model 1qxx was carried out with MOLREP (Vagin \& Teplyakov, 1997) from the $C C P 4$ program package (Collaborative Computational Project, Number 4, 1994). This structure represents the TonB-77 dimer (Koedding et al., 2004), which is very similar to the TonB-86 dimer (Chang et al., 2001). Unfortunately, no useful phase information was obtained using this search model either with or without side-chain atoms. Molecular

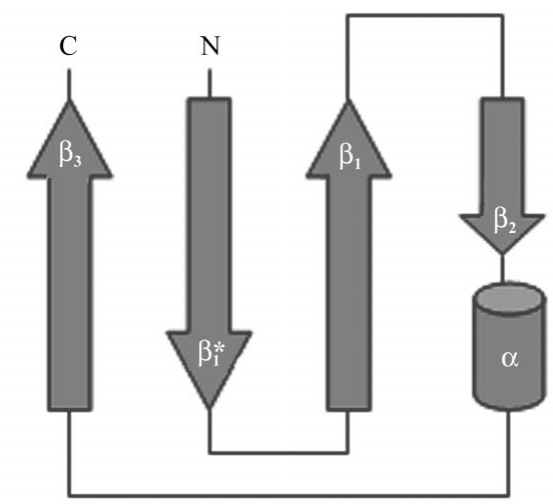

Figure 2

Putative topology model of TonB-92. 
replacement with a model consisting of an isolated monomer of the TonB-77 dimer also failed to give sufficient phase information (Table 2). Additionally, a search model was created based on the putative topology model of TonB-92 (Fig. 2) that we proposed previously for the TonB-96 fragment (Koedding et al., 2004). This model consists of a TonB-77 monomer with an additional $\beta$ strand at the N-terminus ( $\beta_{1}^{*}$ in Fig. 2 ) that might fold between $\beta$-strands 1 and 3. The results of the molecular replacement showed bad packing interactions. Refinement of the data with the program REFMAC5 (Murshudov et al., 1997) failed. The fact that none of these models gave us useful phase information indicates that the structure of TonB-92 may differ significantly from the published Ton-77 and TonB-86 dimers. We are currently working on phase determination by direct phasing methods and we are also trying to crystallize TonB-92 with incorporated selenomethionine.
We thank the staff at the SLS synchrotron beamline for their support.

\section{References}

Bradbeer, C. (1993). J. Bacteriol. 175, 31463150.

Braun, V. \& Herrmann, C. (1993). Mol. Microbiol. 8, 261-268.

Chang, C., Mooser, A., Pluckthun, A. \& Wlodawer, A. (2001). J. Biol. Chem. 276, 27535-27540.

Collaborative Computational Project, Number 4 (1994). Acta Cryst. D50, 760-763.

Diederichs, K. \& Karplus, A. P. (1997). Nature Struct. Biol. 4, 269-275.

Doublié, S. (1997). Methods Enzymol. 276, 523530.

Günter, K. \& Braun, V. (1990). FEBS Lett. 274, 85-88.

Howard, S. P., Herrmann, C., Stratilo, C. W. \& Braun, V. (2001). J. Bacteriol. 183, 58855895.

Jancarik, J. \& Kim, S.-H. (1991). J. Appl. Cryst. 24 409-411.

Kabsch, W. (1993). J. Appl. Cryst. 26, 795-800.
Khursigara, C. M., DeCrescenzo, G., Pawelek, P. D. \& Coulton, J. W. (2004). J. Biol. Chem. 279, 7405-7412.

Koedding, J., Howard, P., Kaufmann, L., Polzer, P., Lustig, A. \& Welte, W. (2004). J. Biol. Chem. 279, 9978-9986.

Larsen, R. A., Thomas, M. G. \& Postle, K. (1999). Mol. Microbiol. 31, 1809-1824.

Larsen, R. A., Wood, G. E. \& Postle, K. (1994). Mol. Microbiol. 12, 857.

Moeck, G. S. \& Letellier, L. (2001). J. Bacteriol. 183, 2755-2764.

Murshudov, G. N., Vagin, A. \& Dodson, E. J. (1997). Acta Cryst. D53, 240-255.

Postle, K. (1993). J. Bioenerg. Biomembr. 25, 591601.

Postle, K. \& Kadner, R. J. (2003). Mol. Microbiol. 49, 869-882.

Postle, K. \& Skare, J. T. (1988). J. Biol. Chem. 263 11000-11007.

Sauter, A., Howard, S. P. \& Braun, V. (2003). J. Bacteriol. 185, 5747-5754.

Vagin, A. \& Teplyakov, A. (1997). J. Appl. Cryst. 30, 1022-1025.

Witty, M., Sanz, C., Shah, A., Grossmann, J. G., Mizuguchi, K., Perham, R. N. \& Luisi, B. (2002). EMBO J. 21, 4207-4218. 